\title{
Optimization of 5G Fronthaul based on Functional Splitting at PHY Layer
}

\author{
Rakibul Islam Rony, Elena Lopez-Aguilera, Eduard Garcia-Villegas \\ Dept. of Network Engineering \\ Universitat Politècnica de Catalunya, Barcelona, Spain \\ Email: rakibul.islam.rony@upc.edu, \{elopez, eduardg\}@entel.upc.edu
}

\begin{abstract}
G is coming with a promise to provide ubiquitous coverage with high data rate availability. To do so, densification of access points to enhance the system capacity is anticipated. For managing such densely populated network, 5G will be employing Centralized Radio Access Network (CRAN), where most of the Radio Access Network (RAN) functionalities are centralized in a central processing unit. This centralization reduces operational costs and eases implementation of advanced technologies, such as, Cooperative multipoint (CoMP) and enhanced inter-cell interference coordination (eICIC), in a cost efficient way. However, CRAN imposes stringent requirements on the fronthaul, i.e. the link connecting access points to the central unit, in terms of capacity and latency. Furthermore, future fronthaul networks are expected to rely on wireless technologies, since wired options are costly, not scalable and not always suitable for all scenarios. Therefore, meeting the expected requirements of fronthaul network utilizing capacity-limited wireless technologies may become an inescapable bottleneck. In this paper, we study different functional splits at the PHY layer in terms of data rate requirements and operational cost, and discuss the combination of different splits aimed at minimizing the overall cost and maximizing the centralization gains, while keeping the capacity requirements below the limit of the fronthaul.
\end{abstract}

\section{INTRODUCTION}

Popularity of mobile devices and data-rich applications are putting high capacity demand on the future wireless networks. Hence, instead of futuristic vision, Fifth Generation (5G) is becoming today's reality [1], and is preparing to meet thousand times system capacity $/ \mathrm{km}^{2}$, hundred times data rate and number of connected devices compared to Long Term Evolution (LTE) [2]. To meet the expected performance, Centralized Radio Access Network (CRAN) is identified as a key enabler, where most of the Radio Access Network (RAN) functionalities are centralized in a Baseband Unit (BBU) and the Access Points (AP), known as Remote Radio Heads (RRH), perform basic Radio Frequency (RF) functionalities. Additionally to the demand of ultra dense network composed of densely deployed APs to serve huge number of users expecting very high data rate in the access network, CRAN will be asking for a very high capacity and low latency fronthaul $(\mathrm{FH})$ network connecting BBU to the RRHs. To tackle the stated challenge, the industry and the academia studied different functional splits, where the distribution of PHY functions between the RRH and the BBU varies. The more functions are given to the $\mathrm{BBU}$, the more flexible is the architecture, but the requirements imposed to the $\mathrm{FH}$ are harder to meet. Figure 1 illustrates the basic architecture of

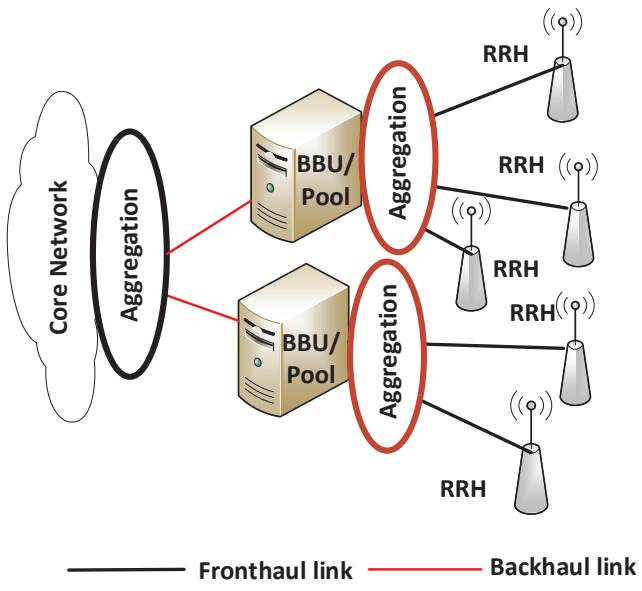

Fig. 1. Basic CRAN architecture.

future CRAN, where the links connecting the BBUs to the subsequent part of the network are referred to as Backhaul (BH).

For the aforementioned FH network, two main specifications, known as Common Public Radio Interface (CPRI) and Open Base Station Architecture Initiative (OBSAI), have been defined. In CRAN, CPRI/OBSAI carries all the Inphase and Quadrature (IQ) data between the RRHs and BBU, requiring huge data rate, and thus limiting the options for FH interfaces. Optical fibre-based wired network provides the highest performance as $\mathrm{BH} / \mathrm{FH}$ option in terms of capacity and latency. Utilizing Time and Wavelength Division Multiplexing (TWDM), 40-Gigabit Passive Optical Network (PON), also known as TWDM PON (ITU-T G.989), has been standardized and is capable of providing $40-80 \mathrm{Gbps}$ of data rate. However, according to [3], only sixteen countries in the world have more than $15 \%$ coverage of Fibre to The Home (FTTH) available. In this scenario, optical fibre based options are costly for new deployments and lack of scalability. Additionally, eight different use cases of 5G service are identified in [4], for which APs are expected to be located in diverse positions, such as top of lamp-posts or mounted on a wall, thus making fibre-based transport network not always a viable option. Hence, wirelessbased approaches are getting more popular since they are cheaper, easier and faster to deploy. In this way, $68 \%$ operators 
consider wireless technologies as a priority element for 5G [1]. With this in mind, in [5], authors anticipated that wireless FH options operating in a higher carrier frequency will be more popular in future practical CRAN, compared to optical fibre based FH networks. However, wireless-based FH/BH shows two major drawbacks: limited capacity and shorter covered distance. Nonetheless, future wireless technologies, such as mmWave E-band (71-76 GHz) (which does not suffer from oxygen absorption, and hence, can cover longer distances) will be capable of offering data rates of $10 \mathrm{Gbps}$ [6]. Additionally, mmWave D-band (141-174.8 GHz), which is still under development, is expected to provide $30-50 \mathrm{Gbps}$ of data rate with a covered distance of more than $1 \mathrm{~km} \mathrm{[1].}$

It is also known that CRAN will be a cost-effective solution compared to the traditional Distributed RAN (DRAN), where APs perform all the RAN functionalities [3] [7]. On the other hand, different functional splits allowing more relaxed $\mathrm{FH}$ requirements can potentially increase the anticipated Capital Expenditure (CAPEX) and Operational Expenditure (OPEX) of future RAN, which might turn out to be a critical issue in the migration towards $5 \mathrm{G}$. Thus, in this article, we analyse the throughput and cost performance of different splits at the PHY layer utilizing wireless options in the FH network. To do so, we first look into the Flexible RAN architecture and how the RAN functionalities can transition between RRH and BBU. Subsequently, we focus on functional splits at the PHY layer and discuss their throughput requirements and cost performance. Next, we provide a solution to offer the best use of the splitting at the PHY layer in a capacity-limited FH network, i.e. not capable to meet full CRAN requirements in all the FH links managed by a common BBU. We present the results, where we find the optimal combination of different functional splits at PHY layer minimizing the OPEX and maximizing the centralization in a scenario to be deployed. This paper is concluded identifying future potentials of this work.

\section{Flexible RAdio Access Network}

Traditionally, in a DRAN approach, Base Stations (BSs)/ evolved Node-Bs (eNBs) perform all the RAN functionalities (e.g. admission control, resource management, medium access control (MAC)) while network management is done centrally. Thus, the backhaul links connecting the eNBs to the Core Network $(\mathrm{CN})$ are less demanding in terms of capacity and latency. However, DRAN lacks performance in terms of resource management, cost and energy efficiency [3].

On the other hand, CRAN's BBUs are responsible for the central resource management of the RRHs connected to it, enabling centralization gains. In CRAN, designing low cost $\mathrm{RRH}$ is easier, and centralized control ensures efficient use of resources utilizing network-wide knowledge. This makes CRAN very cost effective and an attractive option for operators. Additionally, according to extensive simulation results (e.g. [7]), CRAN outperforms DRAN in almost every key performance indicator, i.e. cell-average and cell-edge throughput, spectral and energy efficiency. However, despite the benefits
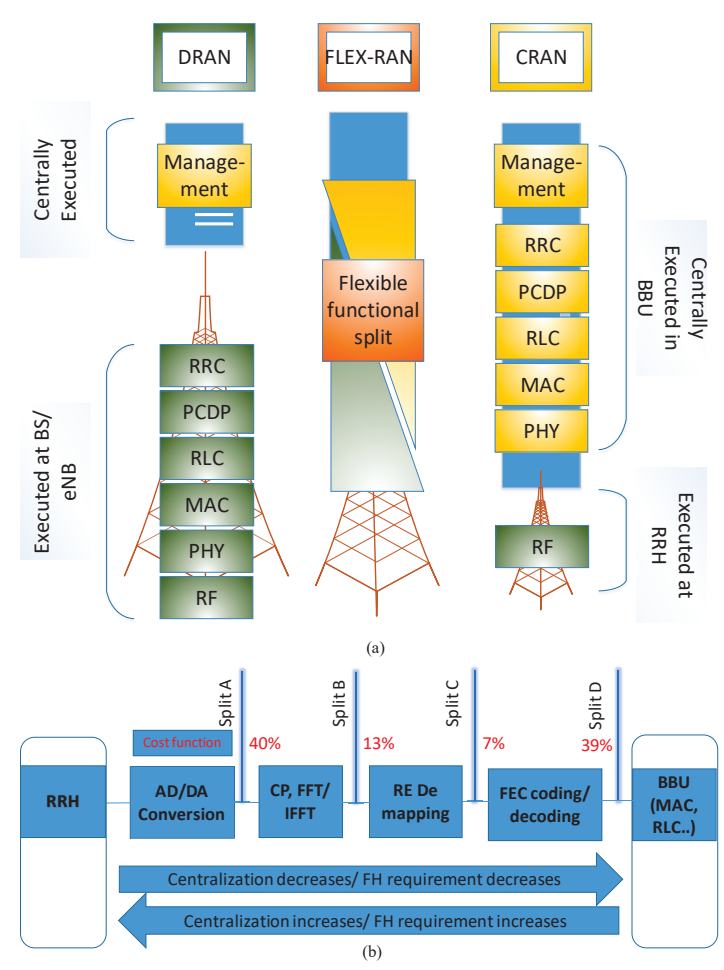

Fig. 2. a) FLEX-RAN, trade-off between CRAN and DRAN; b) Functional splits at PHY layer and corresponding cost distribution [3].

of CRAN, this mechanism demands very high capacity and very a low latency transport network [8].

Between the aforementioned benefits and drawbacks of both approaches (i.e. DRAN and CRAN), a trade-off solution was proposed by iJOIN project as RAN as a Service (RANaaS) [9], which allows Flexible RAN architecture. Flexible RAN (FLEX-RAN), as shown in Figure 2(a), allows the RAN functionalities to transition between fully centralized and fully decentralized architectures on demand, making RAN more flexible, while also relaxing $\mathrm{BH} / \mathrm{FH}$ requirements. The $3 \mathrm{rd}$ Generation Partnership Project (3GPPP) also defines different functional splits between central and distributed unit in TR 38.801 Rel.14.

\section{A. Functional splits at PHY layer}

Functional splits at the PHY layer are a key enabler of the Flexible RAN concept that allows the centralization of upper layers, i.e. MAC, Radio Link Control (RLC), etc, while relaxing the stringent throughput requirements in $\mathrm{FH}$ network. Authors in [3] [6] [10] [11] [12] discuss functional splits at the PHY layer, and the derived trade-off between centralization and FH requirements. As depicted in Figure 2(b), in this work we focus on four potential splitting points at the PHY layer. As in [13], we assume that transfer of the analysis in the Uplink (UL) scenario to the Downlink (DL) scenario is straightforward and, henceforth, we only discuss the UL communication. 
In Split-A, all the PHY layer functionalities along with upper RAN layers are centralized and processed at BBU, resembling the CRAN approach. As mentioned earlier, in this case all the IQ data is forwarded from the RRHs to the $\mathrm{BBU}$ in the UL after Analog to Digital (AD) conversion, and usually, it is referred to as Radio-over-Fibre (RoF), used in CPRI standard [11]. Thus, the FH data rate requirement for this split depends on the number of AD converter chains, sampling frequency, transport overhead and resolution of the time domain quantizer. Hence, $\mathrm{FH}$ data rate requirement for Split-A is static, agnostic to the real traffic scenario and extremely high (4.9 Gbps expected for Sub-6 GHz access technology, and $199.5 \mathrm{Gbps}$ for high mmWave) [10]. Major benefit of this split is that almost no processing in RRH is required, resulting in more cost efficient RRHs. Additionally, even if a fully functional AP acts as RRH with Split-A, the power consumption by the APs decreases considerably since the Digital Unit (DU) in the APs can be totally powered off. Moreover, this split does not restrict any type of centralization, e.g. implemetation of Cooperative multipoint (CoMP), enhanced inter-cell interference coordination (eICIC), which can improve the network performance [12].

In Split-B, the received signal is forwarded from the APs to the BBU in the frequency domain after Fast Fourier Transform (FFT) [11]. Thus, the required data rate is now dependent on the number of active subcarriers, number of symbols per frame, the resolution of frequency domain quantizer and the frame duration [10]. Despite the decreased capacity requirements, this split also has almost no restrictions on the centralization gain, but it imposes additional functionality at the APs, which increases the OPEX of DUs.

Split-C can be considered a more practical split, since the required data rate scales with the actual data traffic in the access network. As depicted in Figure 2(b), the resource mapping/demapping is done locally at the APs, and hence, only the utilized Resource Elements (RE) are forwarded in the UL transmission to the BBU. Required data rate of this split is almost the same as for Split-B with additional dependency on data traffic/RE utilization factor [11]. Hence, in full load, the requirements for Split-C and Split-B are equal.

In Split-D, all the PHY layer functionalities are performed locally at the APs and the upper layer functionalities are centralized. In this split, the required data rate shows dependency on the coding rate and the number of modulation symbols [10], hence relaxing the required data rate further more. This split does not allow joint transmission and reception in CoMP [10], yet the centralization gain from joint scheduling, interference coordination and path management techniques are still achievable [12].

Reflected from the aforementioned discussion, different Radio Access Technologies (RAT) in the access network have different data rate requirements in the FH. Looking towards $5 \mathrm{G}$, reference [14] identifies two promising RATs, i.e. Sub$6 \mathrm{GHz}$ (Carrier frequency (CF) at $3.5 \mathrm{GHz}$ with $100 \mathrm{MHz}$ channel bandwidth (BW)) for Macro Base Stations (MBS) and mmWave ( $\mathrm{CF}$ at $25 \mathrm{GHz}$ with $1 \mathrm{GHz}$ channel $\mathrm{BW}$ ) for

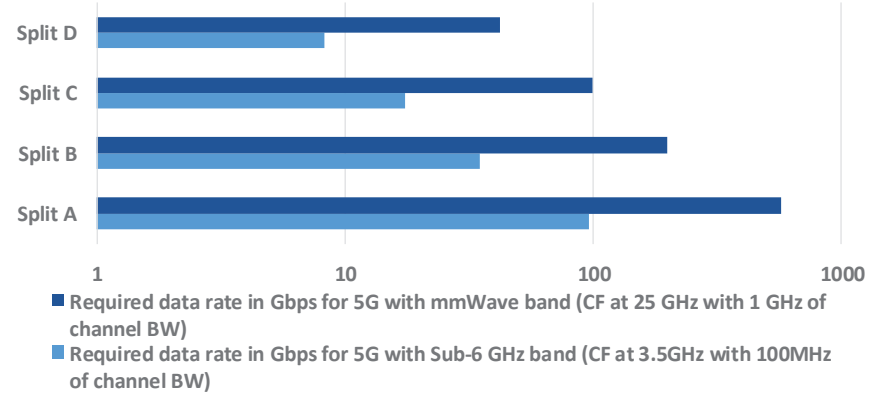

Fig. 3. FH data rate requirement in Gbps for different splits with different technologies considering $50 \%$ of traffic load [10].

Small Cells (SC). Utilizing the equations presented in [10], in Figure 3 we present the corresponding FH requirements for the aforementioned RATs and the different functional splits. Within the highest split point (Split-D) all the PHY layer functions are locally processed, yet upper layer functions can be centralized to have significant impact on the network performance [12]. Hence, the discussed functional splits at PHY layer can be potentially utilized to relax FH requirements and yet achieve certain level of benefits brought by CRAN approach. Moreover, functional splits within the upper layers (e.g. MAC and Radio Resource Control (RRC)) are also possible and can further relax $\mathrm{FH}$ requirements, which will still pose corresponding benefits and challenges [8].

\section{B. Cost distribution of different splits}

As concluded from the previous discussion, utilization of PHY layer splits can relax the stringent requirements of $\mathrm{FH}$ network in future RAN. Nonetheless, with higher splits, centralization decreases, which affects the network performance. Additionally, according to the cost function distribution of different splits presented in [3], with higher splits, the total OPEX (i.e. OPEX of AP + OPEX of BBU) increases. Authors in the aforemetioned work distributed the cost of PHY layer functionalities among different splits as depicted in Figure 2(b) ${ }^{1}$. In this way, OPEX of APs for different splits can be calculated as $\left(A P_{O P E X} \times f(S)\right)$, where $\mathrm{S}$ refers to the split (A,B,C or D), and $A P_{O P E X}$ is the OPEX corresponding to an individual AP. $\mathrm{W}_{\mathrm{s}}$ is the cost function shown in Figure 2(b) (in $\%$ ), so that $f(S)=\sum^{S} W_{\mathrm{s}}$. In other words, $f(S)$ represents the summation of cost functions $\left(\mathrm{W}_{\mathrm{s}}\right)$ of the functionalities run at the AP, e.g. for Split-C, $f(C)=0.4+0.13+0.07$. Similarly, OPEX of BBU also varies for different splits as $\left(B B U_{O P E X} \mathrm{X}\right.$ $(1-f(S))$.

Table I summarizes the OPEX calculation details of BBUs and APs for different splits, as suggested in [3]. Utilizing the presented values, yearly total $\mathrm{OPEX}(€)$ (OPEX of AP + OPEX of BBU) for one MBS and one SC are illustrated in Figure 4. Evidently, utilization of higher splits (i.e. Split-D/C)

\footnotetext{
${ }^{1}$ According to [3], remaining $1 \%$ of the cost belongs to the MAC layer, and it is always included in the BBU's OPEX.
} 
increases the OPEX. Thus, it is both cost and centralization efficient to utilize lower splits (i.e. Split-A/B) at the PHY layer. Additionally, from Figure 4, it is shown that it is more cost efficient to operate MBS with lower splits, since the difference of OPEX between MBS and SC is of one order of magnitude. On the other hand, as illustrated in Figure 3, recall that lower splits ask for higher data rate, which is a big challenge in future wireless-based FH networks.

From the above discussion we can conclude that it is necessary to quantify the trade-off of the different splits at the PHY layer in order to find the optimal combination of splits in a deployment, which minimizes the cost, and maximizes the centralization, while keeping the capacity requirements within the limits.

TABLE I

COST ANALYSIS OF AP AND BBU, THE DEPENDENCY ON PHY LAYER SPLITS [3]

\begin{tabular}{|c|c|}
\hline Parameters & Value \\
\hline $\begin{array}{l}\text { CAPEX } \\
\left(M B S_{C A P E X}\right) \text { and OPEX } \\
\left(M B S_{O P E X}\right) \text { of MBS }\end{array}$ & $\begin{array}{l}53,110 \quad € / \text { year; } 19,775 \\
€ / \text { year }\end{array}$ \\
\hline $\begin{array}{l}\text { CAPEX }\left(S C_{C A P E X}\right) \text { and } \\
\text { OPEX }\left(S C_{O P E X}\right) \text { of SC }\end{array}$ & $7,910 € /$ year; $1,950 € /$ year \\
\hline $\begin{array}{l}\text { OPEX of a MBS } \begin{array}{r}\text { with } \\
\text { functional } \\
\text { MBS }\end{array} \text { splits } \quad \text { (Split- } \\
\text { OPEX }\end{array}$ & $M B S_{O P E X} * f(S) € /$ year \\
\hline $\begin{array}{l}\text { OPEX of a SC with func- } \\
\text { tional splits }\left(\text { Split-SC } C_{O P E X}\right)\end{array}$ & $S C_{O P E X} * f(S) € /$ year \\
\hline $\begin{array}{l}\text { CAPEX of BBU while } \\
\text { centralizing a } \\
\left.M B S_{C A P E X}\right)\end{array}$ & $\begin{array}{l}M B S_{C A P E X} \\
€ / \text { year }\end{array}$ \\
\hline $\begin{array}{l}\text { OPEX of BBU while } \\
\text { centralizing a MBS }(B B U- \\
\left.M B S_{O P E X}\right)\end{array}$ & $\begin{array}{l}\left(B B U-M B S_{C A P E X}\right) / 16.34 \\
€ / \text { year [15] }\end{array}$ \\
\hline $\begin{array}{l}\text { CAPEX of } \text { BBU } \\
\text { centralizing a } \text { SC } \quad(B B U- \\
\left.S C_{C A P E X}\right)\end{array}$ & $S C_{C A P E X} *(1-f(S)) € /$ year \\
\hline $\begin{array}{l}\text { OPEX of } \\
\text { centralizing a }\end{array}$ & $\begin{array}{l}\left(B B U-S C_{C A P E X}\right) / 16.34 \\
€ / \text { year }[15]\end{array}$ \\
\hline
\end{tabular}

\section{Proposed framework and evaluation results}

Flexible RAN, which offers a trade-off between CRAN and DRAN is a stand-up concept making the transport network more realistic and flexible for future mobile networks. In this work, we focus on the PHY layer splits, considering that upper layers are centralized and processed at the BBU. In this section, we evaluate different strategies based on the concept of Flexible RAN, showing that the disscussed splits at the PHY layer truly open an opportunity to make the best use of scarce resources in an efficient way to ensure the maximum achievable centralization including minimum expenditure.

To perform the evaluation, we consider a dense area of $1 \mathrm{~km}^{2}$ served by a number of MBSs and SCs connected to a common $\mathrm{FH}$ aggregator/BBU. Figure 5 illustrates the basic idea of the considered scenario. Also, as mentioned in [5], the future wireless-based FH networks will be capacity constrained. Thus, in this optimization problem, we find the optimal combination of different splits assuming the $\mathrm{FH}$

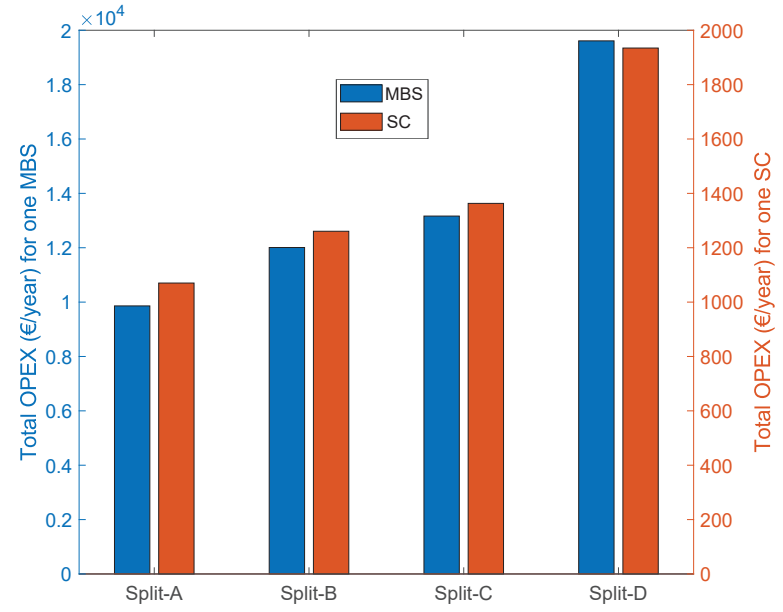

Fig. 4. OPEX (€/year) for different split options, and for one MBS and one SC.

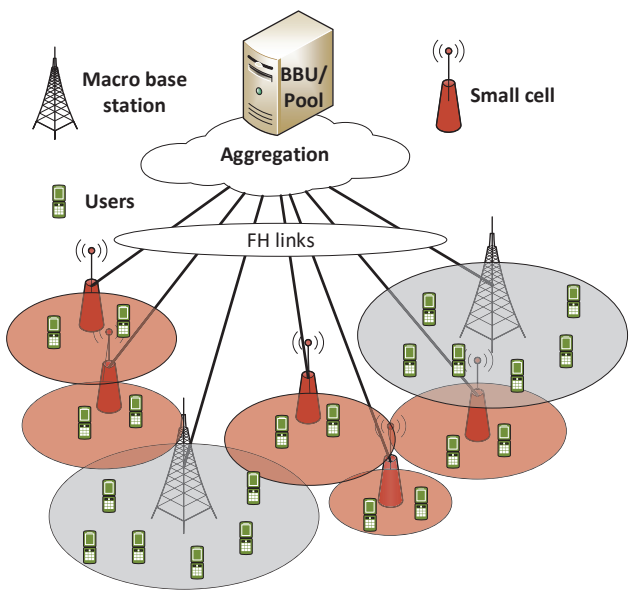

Fig. 5. Considered scenario for evaluation.

aggregator/BBU capacity is limited to a certain percentage of actual CRAN capacity requirements, i.e. capacity required if all the FH links operate in Split-A (capacity required for Split-A x Number of FH links). As an objective function, we consider two different approaches: (1) minimizing the OPEX, where finding the combination of different splits resulting into minimum cost is the objective, and (2) maximizing the centralization, where maximizing the number of $\mathrm{FH}$ links operating in Split-A is prioritized, and subsequently, priority decreases for the higher splits.

We present the benefits of both approaches in different deployments. Table II summarizes the parameters used for the performed evaluation.

Deployment scenario 1 (cf. Table II) considers all the MBSs and SCs operating in Sub-6 GHz band. In this scenario, considering the first approach in which the overall OPEX is minimized, Figure 6 depicts the number of FH links operating in different splits for different level of capacity availability in the FH aggregator/BBU. Utilizing a brute force algorithm, we 
TABLE II

PARAMETERS USED FOR EVALUATION

\begin{tabular}{|c|c|}
\hline Parameters & Value \\
\hline Number of MBSs & 25 \\
\hline Number of SCs & 200 \\
\hline Traffic load in MBS & $100 \%$ \\
\hline Traffic load in SC & $50 \%$ \\
\hline $\begin{array}{l}\text { Available capacity in } \\
\text { BBU/FH aggregator }\end{array}$ & $\begin{array}{l}10 \%-90 \% \text { of the aggre- } \\
\text { gated requirement for } 225 \\
\text { Split-A FH links }\end{array}$ \\
\hline \multicolumn{2}{|c|}{ Deployment scenario 1} \\
\hline \multirow[t]{2}{*}{ RAT } & $\begin{array}{l}\text { MBS: Sub-6 GHz (CF: } 3.5 \\
\text { GHz, BW: } 100 \mathrm{MHz})\end{array}$ \\
\hline & $\begin{array}{l}\text { SC: Sub-6 GHz (CF: } 3.5 \\
\text { GHz, BW: } 100 \mathrm{MHz})\end{array}$ \\
\hline Data requirement for Split-A & 95.8 Gbps (MBS and SC) \\
\hline Data requirement for Split-B & 34.9 Gbps (MBS and SC) \\
\hline \multirow[t]{2}{*}{ Data requirement for Split-C } & $\begin{array}{l}\text { MBS: } 34.9 \text { Gbps with } 100 \% \\
\text { of traffic load }\end{array}$ \\
\hline & $\begin{array}{l}\text { SC: } 17.45 \text { Gbps with } 50 \% \text { of } \\
\text { traffic load }\end{array}$ \\
\hline \multirow[t]{2}{*}{ Data requirement for Split-D } & $\begin{array}{l}\text { MBS: } 16.5 \text { Gbps with } 100 \% \\
\text { of traffic load }\end{array}$ \\
\hline & $\begin{array}{l}\text { SC: } 8.25 \text { Gbps with } 50 \% \text { of } \\
\text { traffic load }\end{array}$ \\
\hline \multicolumn{2}{|c|}{ Deployment scenario 2} \\
\hline \multirow[t]{2}{*}{ RAT } & $\begin{array}{l}\text { MBS: Sub-6 GHz (CF: } 3.5 \\
\text { GHz, BW: } 100 \mathrm{MHz})\end{array}$ \\
\hline & $\begin{array}{l}\text { SC: mmWave (CF: } 25 \mathrm{GHz} \text {, } \\
\text { BW: } 1 \mathrm{GHz})\end{array}$ \\
\hline \multirow{2}{*}{ Data requirement for Split-A } & MBS: $95.8 \mathrm{Gbps}$ \\
\hline & SC: $574.56 \mathrm{Gbps}$ \\
\hline \multirow{2}{*}{ Data requirement for Split-B } & MBS: 34.9 Gbps \\
\hline & SC: $199.18 \mathrm{Gbps}$ \\
\hline \multirow[t]{2}{*}{ Data requirement for Split-C } & $\begin{array}{l}\text { MBS: } 34.9 \text { Gbps with } 100 \% \\
\text { of traffic load }\end{array}$ \\
\hline & $\begin{array}{l}\text { SC: } 99.59 \text { Gbps with } 50 \% \text { of } \\
\text { traffic load }\end{array}$ \\
\hline \multirow[t]{2}{*}{ Data requirement for Split-D } & $\begin{array}{l}\text { MBS: } 16.5 \text { Gbps with } 100 \% \\
\text { of traffic load }\end{array}$ \\
\hline & $\begin{array}{l}\text { SC: } 42.32 \text { Gbps with } 50 \% \text { of } \\
\text { traffic load }\end{array}$ \\
\hline
\end{tabular}

find the smallest $\operatorname{OPEX}(€ /$ year $)$ among all possible combinations of splits. As discussed in the previous section, each MBS contributes more to the operational cost and, therefore, the cost minimization strategy prioritizes Split-A links to MBSs over SCs. It is also beneficial in terms of the network performance, since MBSs are expected to serve twice users of SCs [14] (100\% load for MBSs vs. 50\% load for SCs). With increasing capacity availability in $\mathrm{FH}$ aggregator/BBU, the number of links operating with lower splits, i.e. Split-A/B, rises, and thus, higher centralization is achievable with minimum OPEX. Additionally, for capacity availability equal or higher than $50 \%$, all the links involved in the scenario belong to Split $\mathrm{A}$ and $\mathrm{B}$, which allows almost full centralization gain as discussed in Section II-A. For congested scenarios, e.g. 10\%, $20 \%, 30 \%$ of available capacity, it is still possible to serve all the FH links, even having some of them configured to Split-B, but higher functional splits at PHY layer are necessary, (i.e. Split C/D) to some extent.

Next, we focus on the second optimization approach in

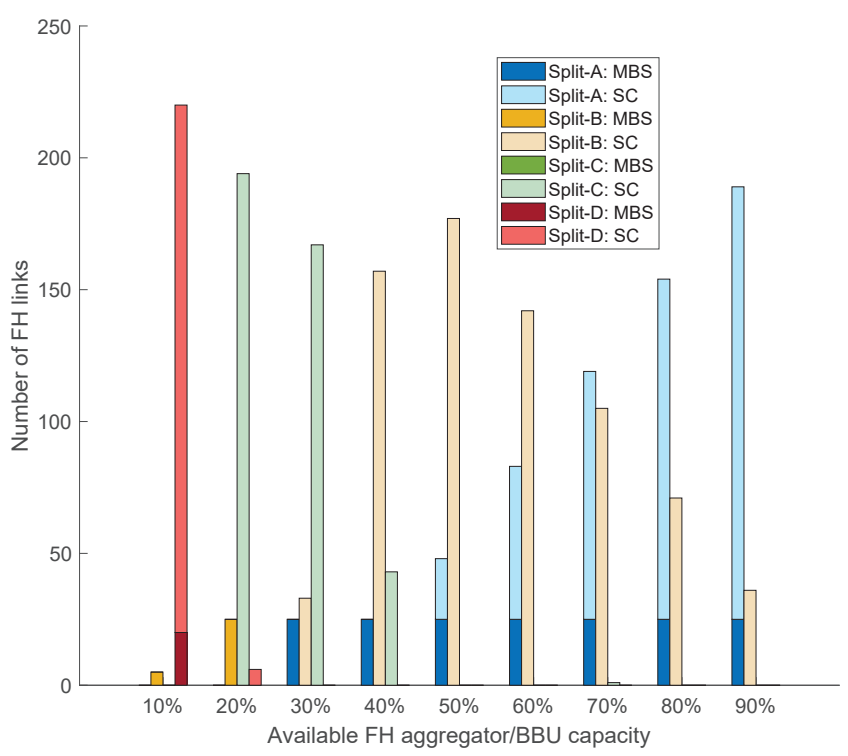

Fig. 6. Scenario 1: Number of FH links utilizing different splits varying with different level of capacity availability in FH aggregator/BBU - minimizing OPEX approach.

which the centralization is maximized. In this way, we find the best combination of links, prioritizing, in the first place, Split-A links for MBSs, and hereafter, Split-B, Split-C and Split-D links for MBSs, in the aforementioned order. Next, we follow the same procedure for SCs. Since, MBSs will be serving higher number of users (100\% load for MBSs vs. $50 \%$ load for SCs), we prioritize MBS links in front of SC links.

Figure 7 illustrates the number of FH links operating in different splits for different level of FH aggregator/BBU capacity, the second optimization approach, in deployment scenario 1. As expected, results show the same trend as the ones presented in Figure 6, since lower splits (i.e. Split-A/B) provide higher centralization, which also leads to lower OPEX. However, there are few differences. For $20 \%$ of available capacity, when minimizing the OPEX, no FH link gets Split-A configuration, and Split-D is only considered for a few of them (Figure 6). On the other hand, under the same capacity conditions, and prioritizing centralization, all 25 MBS FH links operate with Split-A (Figure 7), thus achieving the highest centralization gain, and subsequently, better network performance for the MBS users. However, remaining FH links, i.e. SCs links, have to sacrifice centralization and operate only with Split-D, thus increasing overall OPEX.

Figure 8 illustrates the total OPEX for $225 \mathrm{FH}$ links and the configurations presented in Figures 6 and 7. For the discussed case, i.e. $20 \%$ of available capacity, the difference in cost is clearly visible. Hence, both approaches, i.e. minimizing OPEX and maximizing centralization, can lead to different combination of splits at FH links under some capacity conditions (higher congested scenarios). Mobile operators can decide on the deployment rule to follow based on their particular interests. 


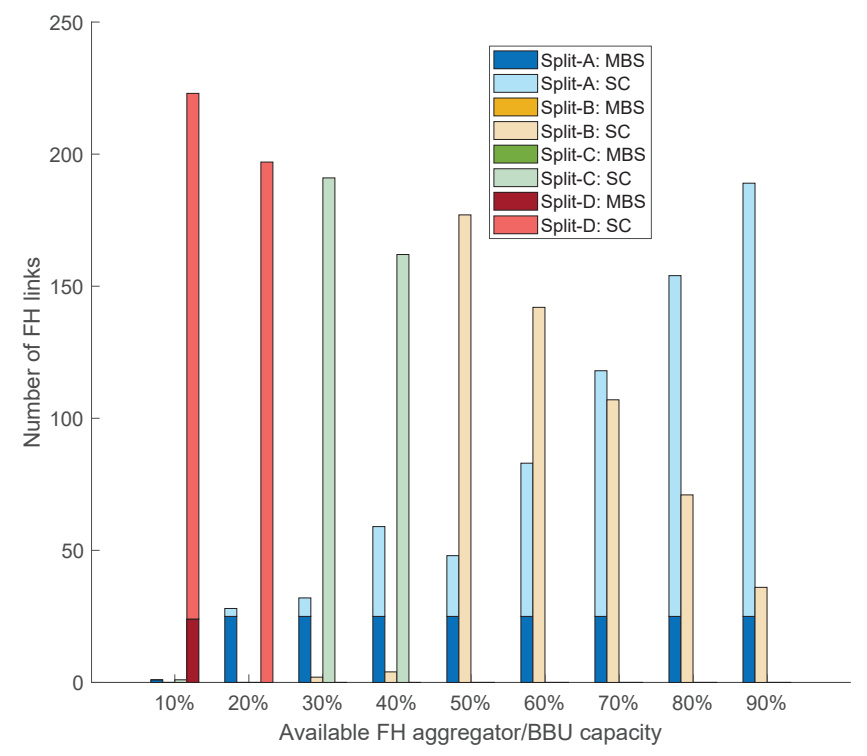

Fig. 7. Scenario 1: Number of FH links utilizing different splits varying with different level of capacity availability in FH aggregator/BBU - maximizing centralization approach.

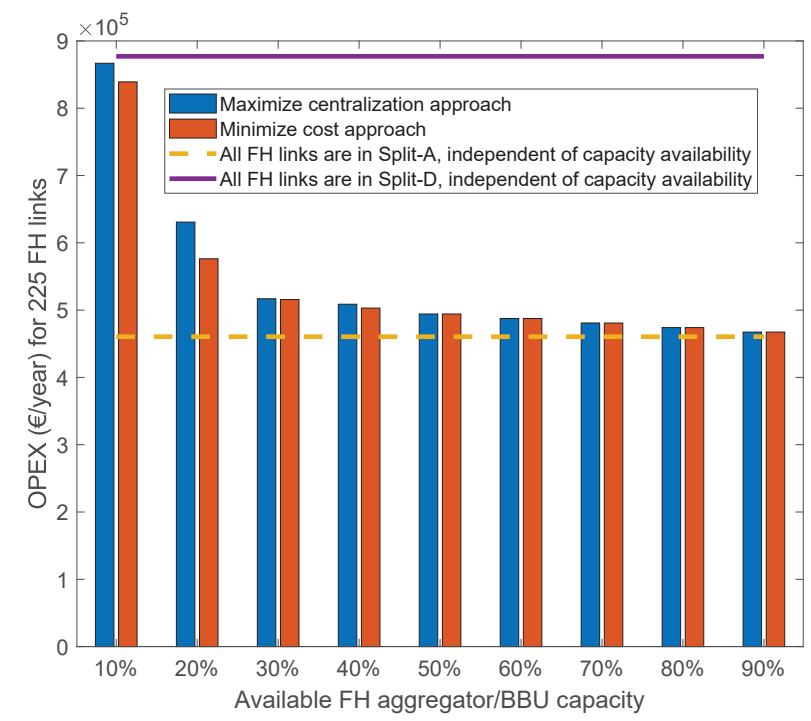

Fig. 8. OPEX (€/year) for the heterogeneous split distributions illustrated in Figures 6 and 7.

We also provide similar evaluation in an heterogeneous scenario, where the MBSs operate in Sub- $6 \mathrm{GHz}$ band and the $\mathrm{SCs}$ in mmWave band as suggested in [14] for 5G (deployment scenario 2, Table II). Thus, in this scenario, we have 25 FH links with Sub-6 GHz and 200 FH links with mmWave. Results are depicted in Figures 9 and 10, and follow the same trend as for scenario 1 in Figures 6 and 7, respectively. In this scenario, all the 25 MBS FH links are capable of achieving full-centralization, i.e. can operate under Split-A, utilizing $10 \%$ of the required capacity at the $\mathrm{FH}$ aggregator/BBU. Note that, in this case, the maximum capacity considered (i.e. capacity required by using Split-A in all links) is larger than in scenario 1 due to the presence of demanding mmWave

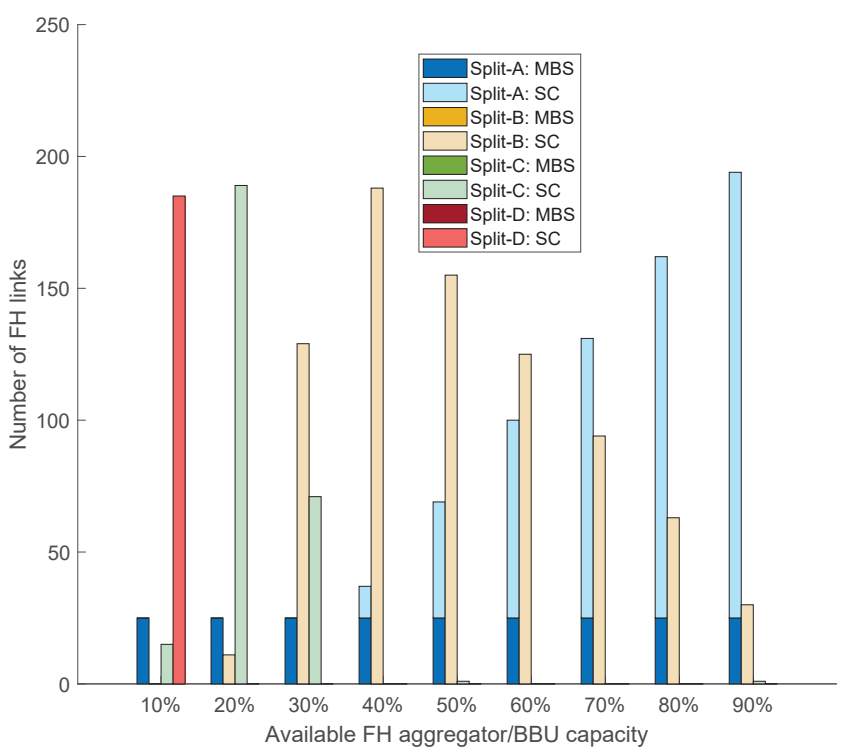

Fig. 9. Scenario 2: Number of FH links utilizing different splits varying with different level of capacity availability in FH aggregator/BBU - minimizing OPEX approach.

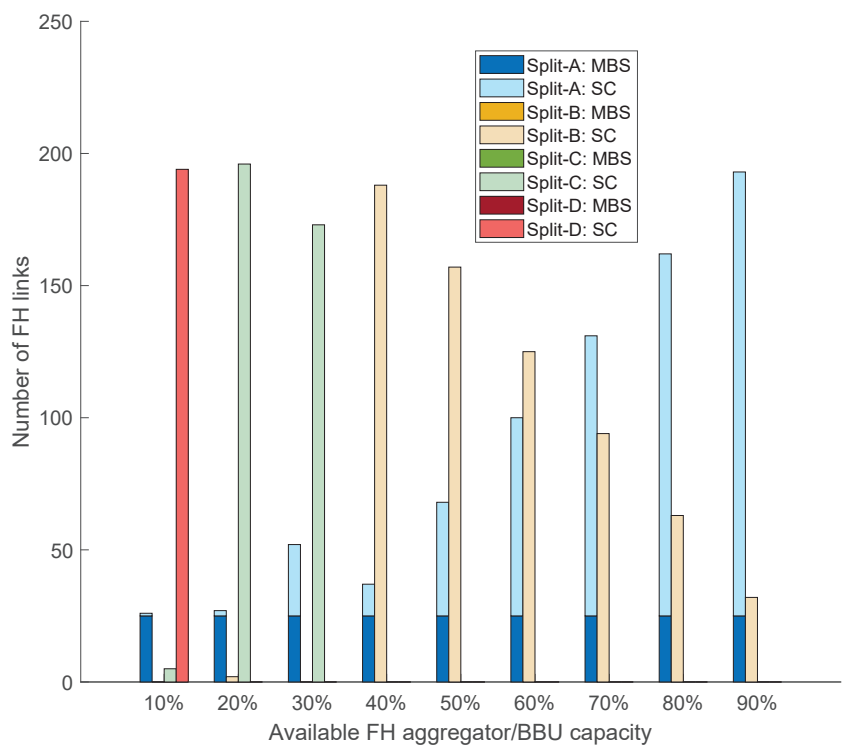

Fig. 10. Scenario 2: Number of FH links utilizing different splits varying with different level of capacity availability in FH aggregator/BBU - maximizing centralization approach.

technology. Figure 11 depicts the OPEX comparison for the configurations presented in Figures 9 and 10, showing the same trend as for scenario 1 .

Despite the different configuration of splits, both approaches show a similar cost when the available capacity is enough to serve most of the MBS links at the lowest splits. In this regard, the cost distribution shown in Figure 2(b) can be finetuned (e.g. weights of different PHY blocks can be slightly redistributed) to prioritize lower/higher splits following the operators preference towards more centralized/distributed $\mathrm{RAN}^{2}$.

\footnotetext{
${ }^{2}$ Optimization of the cost function is out of the scope of this work.
} 
Finally, we compute the FH Capacity Utilization Factor (FCUF), which is defined as the ratio of available vs utilized capacity for different capacity conditions in FH aggregator/BBU. For both the deployment scenarios, i.e. all Sub$6 \mathrm{GHz}$ and mixed Sub- $6 \mathrm{GHz} / \mathrm{mm} W a v e$, and all levels of capacity availability, i.e. 10\%-90\%, the FCUF is higher than $99.6 \%$, for both optimization approaches. Therefore, utilization of different splits at PHY layer to generate an optimal combination for different congestion levels can ensure the best utilization of scarce resources.

\section{CONClusions AND FUture WORK}

This article discuses about future CRAN architecture, benefits and challenges compared to traditional DRAN. It is clear that a trade-off solution between CRAN and DRAN (e.g. FLEX-RAN), makes RAN more flexible. Additionally, functional-splits at PHY layer tackles one of the major challenges of CRAN, i.e. high demand on FH link data rates by flexibly decentralizing PHY layer functionalities towards the AP. Additionally, this approach allows centralization of upper layers, hence, centralization gain is still achievable.

On the other hand, with the higher splits at PHY layer, i.e. more decentralized approach, total OPEX (BBU and AP) increases. Therefore, we presented two techniques to maximize the centralization and minimize the OPEX utilizing different combinations of heterogeneous split deployments. We also presented the combinations for different levels of capacity availability in $\mathrm{FH}$ aggregator/BBU, showing how utilization of splits can still achieve centralization for high priority APs, i.e. MBSs, in capacity-limited scenarios (utilizing 10\% of the required capacity). Additionally, OPEX comparison for different optimization approaches (i.e. minimum OPEX and maximum centralization) are discussed to evaluate the expense due to maximizing the centralization.

In this article we presented the cost analysis of different splits in a per year scenario, assuming the combination of the splits remains static over the year. However, we aim to perform a dynamic analysis, which will be an on-demand basis approach, where such combination of splits can vary with the traffic load conditions in the access network.

\section{ACKNOWLEDGEMENT}

This work has been supported by the EU Horizon 2020 research and innovation programme under grants No. 675806 (5GAuRA) and 762057 (5G-PICTURE).

\section{REFERENCES}

[1] M. Jaber, F. J. Lopez-Martinez, M. A. Imran, A. Sutton, A. Tukmanov, and R. Tafazolli, "Wireless backhaul: Performance modelling and impact on user association for 5g," IEEE Transactions on Wireless Communications, vol. PP, no. 99, pp. 1-1, 2018.

[2] NTT-Docomo, "Docomo 5g white paper," 2014. [Online]. Available: https://www.nttdocomo.co.jp/english/binary/pdf/corporate/ technology/whitepaper $\backslash$ 5g/DOCOMO $\backslash$ _5G $\backslash$ _White $\backslash$ PPaper.pdf

[3] M. Jaber, D. Owens, M. A. Imran, R. Tafazolli, and A. Tukmanov, "A joint backhaul and ran perspective on the benefits of centralised ran functions," in IEEE ICC, May 2016, pp. 226-231.

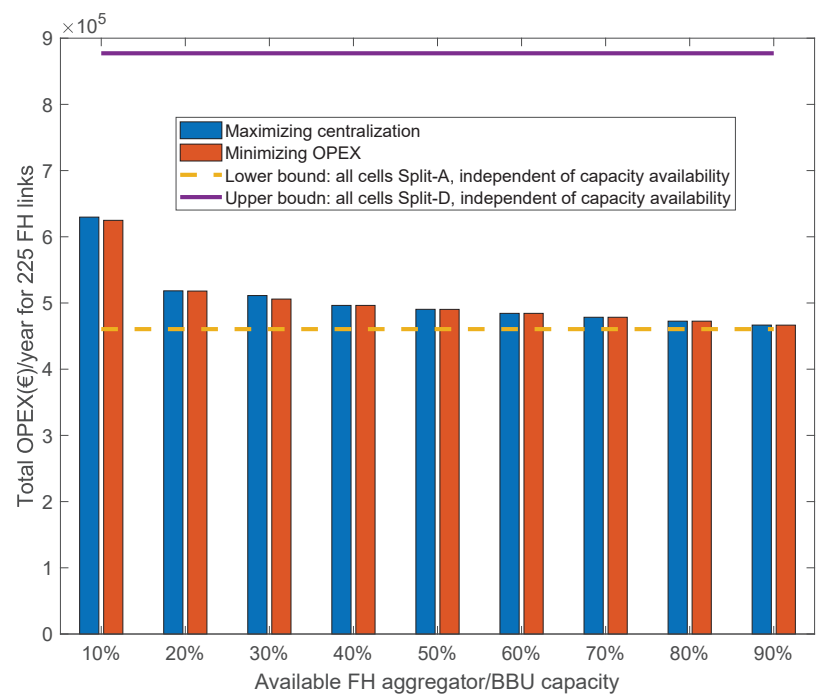

Fig. 11. OPEX (€/year) for the heterogeneous split distributions illustrated in Figures 9 and 10.

[4] NGMN-Alliance, "5g white paper," Next generation mobile networks, white paper, 2015. [Online]. Available: https://www.ngmn.org/fileadmin/ngmn/content/downloads/ Technical/2015/NGMN_5G_White_Paper_V1_0.pdf

[5] M. Peng, C. Wang, V. Lau, and H. V. Poor, "Fronthaul-constrained cloud radio access networks: insights and challenges," IEEE Wireless Communications, vol. 22, no. 2, pp. 152-160, April 2015.

[6] M. Jaber, M. A. Imran, R. Tafazolli, and A. Tukmanov, "5g backhaul challenges and emerging research directions: A survey," IEEE Access, vol. 4, pp. 1743-1766, 2016.

[7] X. Wang, C. Cavdar, L. Wang, M. Tornatore, Y. Zhao, H. S. Chung, H. H. Lee, S. Park, and B. Mukherjee, "Joint allocation of radio and optical resources in virtualized cloud ran with comp," in IEEE GLOBECOM, Dec 2016, pp. 1-6.

[8] A. Maeder, M. Lalam, A. D. Domenico, E. Pateromichelakis, D. Wbben, J. Bartelt, R. Fritzsche, and P. Rost, "Towards a flexible functional split for cloud-ran networks," in $E u C N C$, June 2014, pp. 1-5.

[9] D. Sabella, P. Rost, Y. Sheng, E. Pateromichelakis, U. Salim, P. GuittonOuhamou, M. D. Girolamo, and G. Giuliani, "Ran as a service: Challenges of designing a flexible ran architecture in a cloud-based heterogeneous mobile network," in 2013 Future Network Mobile Summit, July 2013, pp. 1-8.

[10] J. Bartelt, N. Vucic, D. Camps-Mur, E. Garcia-Villegas, I. Demirkol, A. Fehske, M. Grieger, A. Tzanakaki, J. Gutiérrez, E. Grass, G. Lyberopoulos, and G. Fettweis, "5g transport network requirements for the next generation fronthaul interface," EURASIP Journal on Wireless Communications and Networking, vol. 2017, no. 1, p. 89, May 2017. [Online]. Available: https://doi.org/10.1186/s13638-017-0874-7

[11] D. Wubben, P. Rost, J. S. Bartelt, M. Lalam, V. Savin, M. Gorgoglione, A. Dekorsy, and G. Fettweis, "Benefits and impact of cloud computing on $5 \mathrm{~g}$ signal processing: Flexible centralization through cloud-ran," IEEE Signal Processing Magazine, vol. 31, no. 6, pp. 35-44, Nov 2014.

[12] J. Bartelt, P. Rost, D. Wubben, J. Lessmann, B. Melis, and G. Fettweis, "Fronthaul and backhaul requirements of flexibly centralized radio access networks," IEEE Wireless Communications, vol. 22, no. 5, pp. 105-111, October 2015.

[13] U. Dtsch, M. Doll, H. P. Mayer, F. Schaich, J. Segel, and P. Sehier "Quantitative analysis of split base station processing and determination of advantageous architectures for lte," Bell Labs Technical Journal, vol. 18, no. 1, pp. 105-128, June 2013.

[14] METIS-II, "Performance evaluation framework," Deliverable D2.1, no. January, p. 10, 2016. [Online]. Available: https://metis-ii.5g-ppp.eu/ wp-content/uploads/deliverables/METIS-II_D2.1_v1.0.pdf

[15] M. D. Andrade, M. Tornatore, A. Pattavina, A. Hamidian, and K. Grobe, "Cost models for baseband unit (bbu) hotelling: From local to cloud," in IEEE CloudNet, Oct 2015, pp. 201-204. 